Document downloaded from:

http://hdl.handle.net/10251/177804

This paper must be cited as:

Olivares-Sánchez-Mellado, I.; Sanchis Kilders, P. (2020). Enhanced Pockels effect in strained silicon by means of a SiGe/Si/SiGe slot structure. IEEE. 1-2. https://doi.org/10.1109/IPC47351.2020.9252351

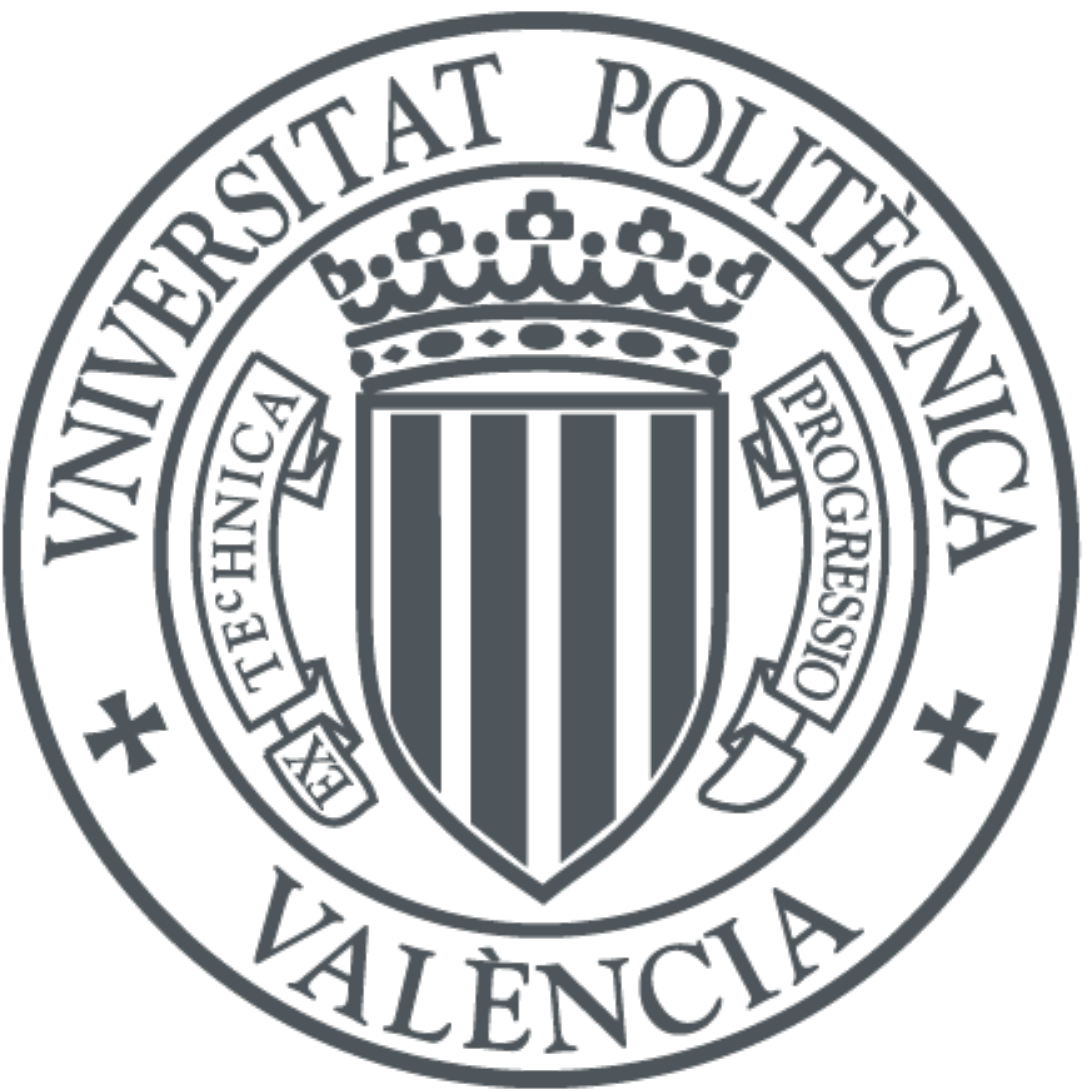

The final publication is available at

https://doi.org/10.1109/IPC47351.2020.9252351

Copyright IEEE

Additional Information 


\title{
Enhanced Pockels effect in strained silicon by means of a $\mathrm{SiGe} / \mathrm{Si} / \mathrm{SiGe}$ slot structure
}

\author{
Irene Olivares* and Pablo Sanchis ${ }^{+}$ \\ Nanophotonics Technology Center, Universitat Politècnica de València, \\ Camino de Vera s/n, 46022 Valencia, Spain \\ E-mail: * irolsan@ntc.upv.es, ${ }^{+}$pabsanki@ntc.upv.es
}

\begin{abstract}
A slot waveguide structure made of a $\mathrm{SiGe/Si/SiGe} \mathrm{heterojunction} \mathrm{is} \mathrm{proposed} \mathrm{to} \mathrm{enhance}$ Pockels effect in strained silicon. The strain is applied via lattice mismatch between layers, while the slot configuration optimizes the overlap between the optical and electric field inside the strained silicon.
\end{abstract}

Keywords—silicon photonics, strained silicon, germanium.

\section{INTRODUCTION}

Strained silicon emerged as a new field of research in photonics more than a decade ago, when a low frequency electro-optic effect was observed in a silicon waveguide covered by a silicon nitride layer with high intrinsic stress [1]. Such results were attributed to the presence of Pockels effect enabled by the strain induced symmetry breaking of the silicon lattice. Although subsequent experimental and theoretical studies revealed that such presence must be weaker than initially thought [2-6], more encouraging results such as the demonstration of second harmonic generation [7] and high frequency modulation [8] in strained silicon waveguides have been also reported since then. In our last work, the effective index change resulting from strain induced Pockels effect was significantly enhanced by using a p-i-n junction [9]. However, the way of applying such strain (mainly using a stressing silicon nitride cover), has not barely changed since the concept was proposed in 2008 [1-9]. This situation contrasts when compared to the microelectronic industry, where a much diverse variety of methods for applying strain can be found, from bridge like structures [10] to lattice mismatched layers [11]. More specifically, the use of epitaxially grown silicongermanium alloys $\left(\mathrm{Si}_{1-\mathrm{x}} \mathrm{Ge}_{\mathrm{x}}\right)$ to apply strain via lattice mismatch is a mature technique employed to tune the electrical and optical properties of silicon and other materials by changing their crystallographic structure. It is commercially and widely employed to enhance, among others, the carrier mobilities in MOSFET and FinFet devices [11] and broadly used to engineer structures such as lightemitting diodes [12] or single-electron quantum devices [13]. Very recently, efficient direct-bandgap emission has been achieved in SiGe alloys [14] marking a milestone in the photonics field and making this material system an ideal platform for combining electronic and photonic devices in the same chip.

In this work, a slot configuration made of a SiGe-Si-SiGe heterojunction is proposed to increase the strain applied to the silicon layer as well as its interaction with the optical mode and electric field. The expected effective index change due to the strain induced Pockels effect exceed those reported to date and approximately double the ones published in our last work [9]. The design of the SiGe-Si-SiGe structure will be described in the next section. Main results are then presented followed by a conclusion.

\section{Proposed SiGe-Si-SiGe SLOT STRUCTURE}

The proposed device consists on a three-layer structure epitaxially grown from a $50 \mathrm{~nm}$ thick (100) silicon SOI wafer. The bottom and top $\mathrm{Si}_{1-\mathrm{x}} \mathrm{Ge}_{\mathrm{x}}$ layers have been chosen to be 200 $\mathrm{nm}$ thick, thus, being fully relaxed. Several variables need to be considered when selecting the Ge composition of the SiGe alloy and the thickness of the silicon layer. In fact, a trade-off between both parameters must be met because, on one side, the lattice mismatch (which rises with a larger Ge concentration) increases the strain applied to the silicon but, on the other, it limits the silicon thickness free of dislocations which can be epitaxially grown on top of the SiGe layer. A final Ge concentration of $x=0.6$ has been chosen, which equals to a lattice mismatch of $2.4 \%$, so that a fully strained silicon layer of around $10 \mathrm{~nm}$ can be epitaxially grown on top of the $\mathrm{Si}_{0.4} \mathrm{Ge}_{0.6}$ alloy [18]. The resulting waveguide structure is depicted in Fig. 1.

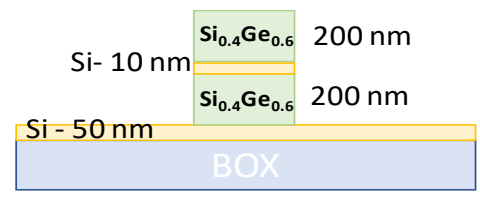

Figure 1. Proposed SiGe-Si-SiGe slot configuration.

\section{PeRformanCE RESUltS}

Once the structure has been proposed, the results for the strain, electric field strength and optical mode confinement have been calculated and are presented in the following sections.

\section{A. Strain applied to the silicon layer}

The strain due to the misfit lattices has been simulated with Silvaco Athena software package [19]. Values around $10^{-2}$ for horizontal (a) and vertical (b) strain components are obtained inside the silicon layer, as can be observed in Fig. 2, which are around one order of magnitude more intense than those of the silicon-silicon nitride waveguide configuration. Moreover, the strain gradients reach values around $10^{5} 1 / \mathrm{m}$, as depicted in Fig. 2 for the (c) $\frac{\partial \varepsilon_{x x}}{\partial x}$ and (d) $\frac{\partial \varepsilon_{x x}}{\partial y}$.
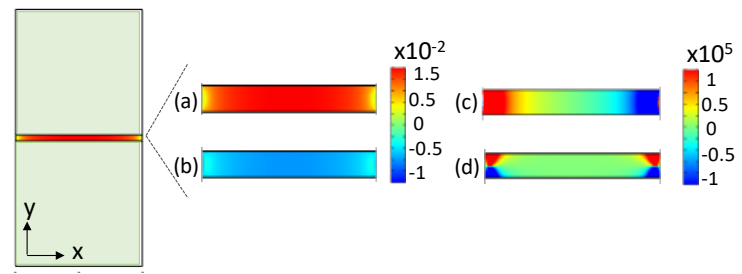

Figure 2. Contour plot of the (a) $\varepsilon_{x x}$ and (b) $\varepsilon_{y y}$ strain components and the (c) horizontal $\frac{\partial \varepsilon_{\mathrm{xx}}}{\partial x}$ and (d) vertical $\frac{\partial \varepsilon_{\mathrm{xx}}}{\partial y}$ strain gradients for $\varepsilon_{\mathrm{xx}}$ in the silicon layer. 


\section{B. Electric field strength inside the strained silicon layer}

To simulate the electrical behavior of the device, the correct values for the $\mathrm{Si}_{1-\mathrm{x}} \mathrm{Ge}_{\mathrm{x}}$ alloy must be considered. Most of optical and electrical parameters follow a linear relationship with concentration, which is the case of the band gap and dielectric constant [17]. Therefore, they have been obtained by interpolating from pure $\mathrm{Si}$ and $\mathrm{Ge}$ values. Table 1 summarizes the parameters used in the simulations.

TABLE I. SI AND $\mathrm{SI}_{1-\mathrm{x}} \mathrm{GE}_{\mathrm{x}}$ MATERIAL PARAMETER VALUES

\begin{tabular}{|l|c|c|c|}
\hline & parameter & units & value \\
\hline \multirow{4}{*}{ Silicon } & Refractive index & & 3.477 \\
\cline { 2 - 4 } & Band Gap & $\mathrm{eV}$ & 1.1 \\
\cline { 2 - 4 } & Electron affinity & $\mathrm{eV}$ & 4.05 \\
\cline { 2 - 4 } & Dielectric constant & & 11.7 \\
\hline \multirow{4}{*}{$\mathrm{Si}_{0.4} \mathrm{Ge}_{0.6}$} & Refractive index & & 3.858 \\
\cline { 2 - 4 } & Band Gap & $\mathrm{eV}$ & 0.84 \\
\cline { 2 - 4 } & Electron affinity & $\mathrm{eV}$ & 4 \\
\cline { 2 - 4 } & Dielectric constant & & 14.4 \\
\hline
\end{tabular}

To obtain the results, the voltage $\left(\mathrm{V}_{\mathrm{g}}\right)$ has been applied to the top SiGe layer while the bottom one has been grounded. The resulting electric field in the whole structure for an applied voltage of $-5 \mathrm{~V}$ is shown in Fig. 3 (a) with the (b) horizontal and (c) vertical components inside the silicon nitride layer. As it can be observed in the images, the heterojunction acts by concentrating the electric field inside the silicon, which contributes to enhance Pockels effect due to its linear relationship with this variable.

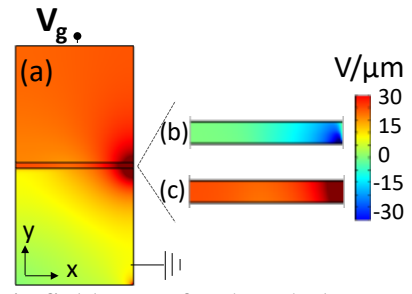

Figure 3. (a) Electric field norm for the whole structure and (b) $E_{x}$ and (c) $E_{y}$ components in the strained silicon layer at $-5 \mathrm{~V}$.

\section{Strain induced effective index change.}

As a result of the low silicon thickness and the small index contrast between $\mathrm{Si}$ and $\mathrm{SiGe}$ (see values of refractive indexes at $1.55 \mathrm{um}$ in Table 1), the guided optical mode is a hybrid between that of a SiGe strip waveguide and the optical mode of the slot structure. Figure 4 (a) shows a contour plot of the optical mode, where it is possible to observe the higher field confinement inside the strained silicon area, also shown in the inset. Using the bond orbital model [5] with the experimental parameters reported in [6], the effective index change has been calculated for the designed structure. Figure 4 (b) shows the results as a function of the applied voltage, where a maximum value of $\sim 2 \cdot 10^{-5}$ is achieved for a voltage sweep between $\pm 5 \mathrm{~V}$. This result improves by around a factor of two the expected effective index change values for Pockels effect compared to our last work [9] and, by a factor of around 400 those expected for the usual silicon-silicon nitride waveguide configuration.
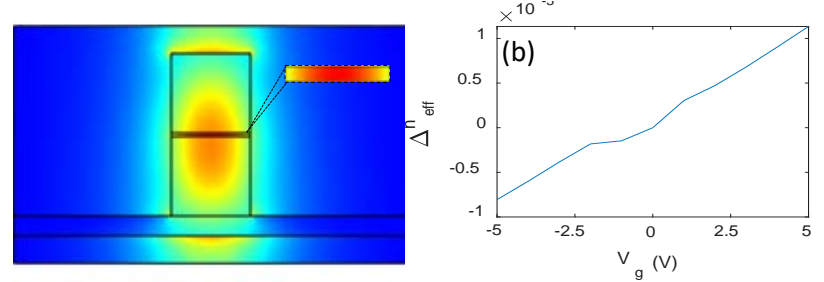

Figure 4. (a) Hybrid optical mode and (b) $\Delta \mathrm{n}_{\text {eff }}$ as a function of $\mathrm{V}_{\mathrm{g}}$.

\section{CONCLUSIONS}

The use of a Si/SiGe slot-based structure to enhance the silicon strain and mode overlap is proposed. Thanks to the designed SiGe-Si-SiGe structure, a strong electric field is achieved inside the silicon, highly strained due to the lattice mismatch between both materials. Furthermore, thanks to the hybrid slot optical mode, the overlap between strain, electric field and optical field is significantly enhanced, leading to an effective index change with values exceeding those reported to date in the literature. The Si/SiGe material system offers a mature technique for applying strain to break the symmetry of silicon. In fact, by engineering the waveguide structure either by growing several $\mathrm{Si} / \mathrm{SiGe}$ layers in its core or by using a graded $\mathrm{Si}_{1-\mathrm{x}} \mathrm{Ge}_{\mathrm{x}}$ as the guiding material, strong stress gradients with high optical overlap could be obtained, offering a path to achieve practical values for strain induced Pockels effect.

\section{ACKNOWLEDGMENT}

Funding from projects TEC2016-76849 (MINECO /FEDER, UE) and PROMETEO/2019/123 (Generalitat Valenciana) is acknowledged. Irene Olivares acknowledges the UPV for funding her research staff training (FPI) grant.

\section{REFERENCES}

[1] R. S. Jacobsen et al., "Strained silicon as a new electro-optic material." Nature, vol. 441, pp. 199-202, 2006.

[2] S. S. Azadeh, F. Merget, M. P. Nezhad, and J. Witzens, "On the measurement of the Pockels effect in strained silicon.” Opt. Lett., vol. 40, pp. 1877-1880, 2015.

[3] M. Borghi, et al, "Homodyne Detection of Free Carrier Induced Electro-Optic Modulation in Strained Silicon Resonators.” J. Light. Technol., vol. 34, pp. 5657-5668, 2016.

[4] I. Olivares, T. Angelova, and P. Sanchis, “On the influence of interface charging dynamics and stressing conditions in strained silicon devices.” Sci. Rep., vol. 7, p. 7241, 2017.

[5] P. Damas, D. Marris-Morini, E. Cassan, and L. Vivien, "Bond orbital description of the strain-induced second-order optical susceptibility in silicon.” Phys. Rev. B, 93(16), 165208 (2016).

[6] P. Damas et al., "Comprehensive description of the electro-optic effects in strained silicon waveguides”. J. Appl. Phys. 122(15), 153105 (2017).

[7] M. Cazzanelli et al, "Second-harmonic generation in silicon waveguides strained by silicon nitride.” Nat. Mater. 11(2),148-154 (2012)

[8] M. Berciano et al. "Fast linear electro-optic effect in a centrosymmetric semiconductor” Communications Physics, 1(1), 64 (2018)

[9] I. Olivares, J. Parra, A. Brimont and P. Sanchis, "Enhancing Pockels effect in strained silicon waveguides” Opt. Express, vol. 27, pp. 2688226892, 2019.

[10] R.A. Minamisawa et al., "Top-down fabricated silicon nanowires under tensile elastic strain up to 4.5\%”, Nat. comm., vol 3, pp.1-6, Oct. 2012.

[11] C. K. Maiti, \& T.K. Maiti, Strain-Engineered MOSFETs, 1st ed., Boca Raton: CRC Press, 2013

[12] D. D. Cannon et al. "Germanium-rich silicon-germanium films epitaxially grown by ultrahigh vacuum chemical-vapor deposition directly on silicon substrates”, Appl. Phys. Lett., vol. 91, p. 252111, Nov. 2007.

[13] S. Goswami, et al. Controllable Valley Splitting in Silicon Quantum Devices. Nat. Phys. 3, 41-45 (2007).

[14] E. M. T. Fadaly et al., "Direct-bandgap emission from hexagonal Ge and SiGe alloys”, Nature, vol. 580, pp. 205-209, April 2020

[18] G. abstreiter, K. eberl, E. Friess, W. Wegscheider and R. Zachai, "Silicon/germanium strained layer superlattices", J. Cryst. Growth, vol. 95, pp. 431- 438, 1989.

[19] www.silvaco.com

[20] Schaffler F et al., "Properties of Advanced SemiconductorMaterials GaN, AlN, InN, BN, SiC, SiGe”. Eds. Levinshtein M.E., John Wiley \& Sons, Inc., New York, 2001, pp. 149-188 